\title{
Defining the phenotype in an autosomal recessive cutis laxa syndrome with a combined congenital defect of glycosylation
}

E Morava*,1 $^{\star 1}$ DJ Lefeber ${ }^{2}$, Z Urban $^{3}$, L de Meirleir ${ }^{4}$, P Meinecke ${ }^{5}$, G Gillessen Kaesbach ${ }^{6}$, $\mathrm{J} \mathrm{Sykut-Cegielska}^{7}, \mathrm{M}$ Adamowicz ${ }^{7}$, I Salafsky ${ }^{8,12}$, J Ranells ${ }^{9}$, E Lemyre ${ }^{10}$, J van Reeuwijk ${ }^{11}$, HG Brunner ${ }^{11}$ and RA Wevers ${ }^{2}$

${ }^{1}$ Department of Pediatrics, Radboud University Nijmegen Medical Centre, Nijmegen, The Netherlands; ${ }^{2}$ Laboratory of Pediatrics and Neurology, Radboud University Nijmegen Medical Centre, Nijmegen, The Netherlands; ${ }^{3}$ Department of Pediatrics and Genetics, Washington University, St Louis, MO, USA; ${ }^{4}$ Department of Human Genetics, University of Brussels, Brussels, Belgium; ${ }^{5}$ Department of Medical Genetics, Altona Children's Hospital, University Clinics HamburgEppendorf, Hamburg, Germany; ${ }^{6}$ Institut für Humangenetik Lübeck, Universitätsklinikum Schleswig-Holstein, Lübeck, Germany; ${ }^{7}$ Department of Metabolic and Endocrine Disorders, The Children's Memorial Health Institute, Warsaw, Poland; ${ }^{8}$ Department of Genetics, Shriner's Hospital for Children, Chicago, IL, USA; ${ }^{9}$ Department of Human Genetics, Regional Genetics Program, University of South Florida, Tampa, FL, USA; ${ }^{10}$ Laboratoire de Cytogénétique Prénatale, Service de Génétique Médicale, CHU Sainte-Justine, Montréal, Québec, Canada; ${ }^{11}$ Department of Human Genetics, Radboud University Nijmegen Medical Centre, Nijmegen, The Netherlands; ${ }^{12}$ Northwestern Medical School, Chicago, IL, USA

Autosomal recessive cutis laxa is a genetically heterogeneous condition. Its molecular basis is largely unknown. Recently, a combined disorder of $\mathrm{N}$ - and O-linked glycosylation was described in children with congenital cutis laxa in association with severe central nervous system involvement, brain migration defects, seizures and hearing loss. We report on seven additional patients with similar clinical features in combination with congenital disorder of glycosylation type Ilx. On the basis of phenotype in 10 patients, we define an autosomal recessive cutis laxa syndrome. The patients have a complex phenotype of neonatal cutis laxa, transient feeding intolerance, late closure of the fontanel, characteristic facial features including down-slanting palpebral fissures, short nose and small mouth, and developmental delay. There is a variable degree of the central nervous system involvement and variable systemic presentation. The biochemical analysis using transferrin isoelectric focusing gives false negative results in some of the youngest patients. Analysis of the apolipoprotein C-III isoelectric focusing, however, is diagnostic in all cases.

European Journal of Human Genetics (2008) 16, 28-35; doi:10.1038/sj.ejhg.5201947; published online 31 October 2007

Keywords: apolipoprotein C-III isofocusing; autosomal recessive cutis laxa; congenital defects of glycosylation; O-glycosylation; CDG type II $\mathrm{x}$

\footnotetext{
*Correspondence: Dr E Morava, Department of Pediatrics, Radboud University Nijmegen Medical Centre, PO Box 9101, Nijmegen 6500 HB, The Netherlands.

Tel: + 3124361 9470; Fax: + 3124361 1600;

E-mail: E.Morava@cukz.umcn.nl

Received 29 June 2007; revised 4 September 2007; accepted 14 September 2007; published online 31 October 2007
}

Introduction

Autosomal recessive cutis laxa (ARCL; MIM 219200, 219100 ) is a genetically heterogeneous condition presenting in the newborn with loose, redundant skin folds, decreased elasticity of the skin and generalized connective tissue involvement. Abnormal elastic fiber structure and a 
significantly decreased amount of elastin are diagnostic findings by skin histology. ${ }^{1,2}$ Fibulin 4 and 5 mutations have been found in some patients with ARCL. ${ }^{3,4}$

The unique association of generalized cutis laxa, developmental and growth delay, joint and skeletal system involvement, characteristic facial features, progressive microcephaly and late closure of the fontanel has been previously described in a few children. ${ }^{2,4-6}$ Congenital defects of glycosylation (CDG) are a group of phenotypically diverse multisystemic disorders caused by various enzyme defects of the $\mathrm{N}$ - (and O-linked) glycan synthesis. In some of these patients, the underlying enzyme deficiency is not yet discovered. The classification of CDG type $x$ (MIM 212067) can be used in cases with an unknown enzyme defect in N-glycosylation.

A combined disorder of the $\mathrm{N}$ - and O-linked glycosylation (congenital disorder of glycosylation type IIx $^{7}$ ) was detected in some patients with cutis laxa in combination with severe central nervous system involvement, intellectual impairment, brain migration defects, seizures and hearing $\operatorname{loss}^{8-10}$ using transferrin isoelectric focusing (TIEF) and ApoC-III isoelectric focusing (IEF) methods. ${ }^{11}$

Abnormal brain development and neuronal migration anomalies are well-known features in Walker-Warburg syndrome and muscle-eye-brain disease, caused by congenital defects in O-linked glycosylation of alpha-dystroglycan. Children diagnosed with the Walker-Warburg syndrome and muscle-eye-brain disease spectrum, however, do not have N-linked glycosylation abnormalities, and they do not express the defective O-linked glycosylation in blood. Connective tissue involvement is not part of the phenotype. ${ }^{12-14}$

\section{Patients and methods}

Following our initial description of three patients with severe generalized cutis laxa, skeletal, neuromuscular and central nervous system involvement, mental retardation and a combined deficiency in the biosynthesis of $\mathrm{N}$ - and O-linked glycans, we additionally evaluated seven children presenting with a clinical phenotype of generalized cutis laxa at birth. All patients were born at term, except for patient 7 (born at 35 weeks of gestation). Only in patient 7 was the family history positive for two early miscarriages. Patients 1, 2, 4 and 5 were offspring from consanguineous marriages. No abnormalities were observed during the pregnancy, except for oligohydramnios in patient 5, who was born by cesarean section. All patients, except for patients 2 and 5, had normal growth parameters at birth. Congenital microcephaly was observed in patients $2,3,5$ and 7. No corneal abnormality, corneal dryness or cataract was found in any of the patients by eye examination. Gastrointestinal diverticulae were not suspected but were also not directly ruled out in the children. None of the patients had symptoms of lung disease, chronic diarrhea, vocal cord laxity, hoarse voice, vascular anomalies, hernias, gastrointestinal anomalies, vesico-uretral reflux or cardiac defects. Only patient 7 had a complex cardiac anomaly. Patients 3 and 5 had congenital bilateral hip dislocations. During the variable follow-up period, none of the patients developed spasticity or dystonia.

\section{Clinical evaluation}

All children underwent ophthalmological and audiological investigations BAEP, EEG, cranial MRI and a cranial and chest X-ray. Patient 1 had a cranial CT. Skin biopsy was performed in patients 1, 2 and 3. Abdominal ultrasound was performed in patients 5 and 7 , and echocardiogram was performed in patients 2, 3, 5, 6 and 7. Development was evaluated by age-appropriate testing modalities: below 4 years of age by the physiotherapist and psychologist, using the Bailey's developmental scale, and above 4 years of age by the psychologist with the age-appropriate modifications of the Wechsler Intelligence Scale for Children III. The clinical findings and results of the investigations are shown in Table 1.

\section{Laboratory evaluation}

All patients underwent routine laboratory tests, including renal and liver function tests, hematology, serum proteins, immunoglobulins, lactic acid, glucose, blood gas, electrolytes, serum calcium, phosphate, alkaline phosphatase, copper, coagulation studies, endocrine evaluation (ACTH, cortisol, GH, IGF, TSH, freeT4) and metabolic studies. Lysosomal enzyme studies in blood and serum, serum amino acids, acyl carnitine profile, urine organic acids and urine thin layer chromatography were performed in patients 1, 2, 3 and 5; serum amino acids, acyl-carnitine profile, VLCFA and phytanic acid in patient 7 . Transferrin and apolipoprotein C-III (ApoC-III) IEF was performed in all patients. ${ }^{8,15,16}$

\section{Patients}

Patient 1 (Figure 1a and b) was the first female child of healthy parents of Belgian origin, who were second cousins (Figure 3a), with a birth weight of $2400 \mathrm{~g}$ (-2 SD). Generalized, severe cutis laxa, dry skin, sparse hair, normocephaly (OFC: $33 \mathrm{~cm}-1 \mathrm{SD}$ ), large anterior fontanel $(5 \times 6 \mathrm{~cm})$, midfacial hypoplasia, anteverted nares, long philtrum, down-slanting palpebral fissures, strabismus, hyperflexible joints, muscle hypotonia and severe feeding difficulties were noted after birth. Feeding problems resolved in a few months and muscle strength improved significantly in the first 2 years. She started to walk at 18 months. The patient also showed a continuous improvement in her skin disease but developed severe caries, brittle hair and myopia (Figure 1c, 1-o). At the age of 11 years, she had age-appropriate height $(135 \mathrm{~cm}:-1 \mathrm{SD})$ and weight (26 kg: $-1 \mathrm{SD}$ ). Skeletal survey was normal. Her 
Table 1 Clinical features and laboratory findings in 10 patients with cutis laxa and CDG type IIx

Clinical features

Consanguinity

Normal birth weight

Congenital microcephaly

Large anterior fontanel

Dysmorphic features

Muscle hypotonia

Developmental delay

Mental retardation

Seizures

Persistent microcephaly

Partial pachygyria (MRI)

Congenital joint anomaly

Hyperelastic joints

Strabismus

Myopia/hypermetropia

Urogenital anomalies

Clinical improvement

Failure to thrive

Liver involvement

Coagulation anomalies

Abnormal TIEF screening

Abnormal ApoC-III screening
Patient 1 Patient 2 Patient 3 Patient 4 Patient 5 Patient 6 Patient 7 *Patient 8 *Patient 9 *Patient 10

$\begin{array}{lll}+ & + & - \\ + & - & + \\ - & + & + \\ + & + & + \\ + & + & + \\ + & + & + \\ + & + & + \\ + & + & + \\ - & - & - \\ - & + & + \\ - & - & + \\ - & - & - \\ + & + & + \\ - & + & - \\ + & - & - \\ - & - & - \\ + & + & + \\ - & - & + \\ + & + & - \\ - & - & + \\ + & + & + \\ + & + & +\end{array}$

+
+
-
+
+
+
+
+
-
-
-
+
+
-
+
-
+
-
-
-
+
+

+ , clinical feature is present; - , feature is absent; NA, feature is not analyzed.

*Morava et al. , $^{\text {, }}$

head circumference is relatively smaller $(51 \mathrm{~cm}:-1.5 \mathrm{SD})$. She has no feeding problems or joint problems. She has a sensitive, dry skin but there is no apparent cutis laxa (Figure 1d and o). Her IQ is 76.

Patient 2 was the first female child of healthy parents from Kurdistan who were first cousins (Figure 3b). Birth weight was $2009 \mathrm{~g}(-3 \mathrm{SD})$ and length $48 \mathrm{~cm}$ (-2 SD). Generalized cutis laxa, proportionate microcephaly (OFC: $30.8 \mathrm{~cm}$ : $-3 \mathrm{SD})$, large fontanel $(4.5 \times 3 \mathrm{~cm})$, brachycephaly, flat face, midfacial hypoplasia, blue sclerae, strabismus, anteverted nares, long philtrum, down-slanting palpebral fissures and hyperflexible joints were noted at birth (Figure $1 \mathrm{e}-\mathrm{g}$ ). The neonate had transient feeding problems and failure to thrive. At the age of 6 months, she had a normal stature $(69 \mathrm{~cm}:-1.5 \mathrm{SD})$ but low body weight (6.9 kg: -2 SD) and mild microcephaly ( -2 SD). Her motor development was delayed; she started to walk at 16 months. At the age of 16 months, she had no swallowing or feeding problems, no hypotonia and had a mean developmental quotient of $74 \%$, with a more obvious speech delay (by bilanguality she uses five words). Skeletal survey was normal, but there is a decreased bone mineralization. Her fontanel is still open $(3 \times 2 \mathrm{~cm})$ and she has significant hyperlaxity of the joints and generalized cutis laxa.

Patient 3 was the first female child of healthy Polish unrelated parents. Normal weight (2940 g: -1.5 SD), generalized cutis laxa, muscle hypotonia, microcephaly $(30.5 \mathrm{~cm}:-2.5 \mathrm{SD})$, large anterior fontanel $(5 \times 7 \mathrm{~cm})$, flat face, midfacial hypoplasia, short nose, anteverted nares, down-slanting palpebral fissures, low set ears, strabismus, thin lips, wide set nipples and anteposition of the anus were noted at birth. Feeding problems persisted in the first 14 months of life. At the age of 22 months, the child eats normally. She shows a delayed growth (length and weight are both $<-2$ SD), microcephaly $(44.3 \mathrm{~cm}:<3 \mathrm{SD})$ with the fontanel that was closed at 16 months of age, mild-tomoderate developmental delay (she can only walk with support and use five words), and she demonstrates a significant muscle hypotonia and cutis laxa.

Patient 4 was the second male child of healthy HispanicAmerican parents who were third cousins once removed (Figure 3c). He had age-appropriate growth parameters, generalized cutis laxa, pectus excavatum, bilateral hip dislocation, joint laxity, pes planus, severe hypotonia and large fontanel. Motor development was mildly delayed. He had recurrent pneumonia. At the age of 4 years, there are no apparent feeding problems. There is severe caries and a continuous improvement of the skin disease. He has a small head circumference $(47 \mathrm{~cm}$ : -2 SD) and a normal stature with age-appropriate body weight.

Patient 5 was the first child of healthy parents, originating from Kurdistan, who were first cousins (Figure 3d). The newborn girl had growth parameters below the -2 SD (length of $45 \mathrm{~cm}$; weight of $2360 \mathrm{~g}$ ), including microcephaly (OFC $31 \mathrm{~cm}$ : <-2SD). Generalized wrinkly skin (Figure 2c and d), abnormal fat distribution with lipodystrophy of the buttocks, and the proximal extremities and fat pads of the hips (Figure 2c-e) and bilateral hip dislocation were noted. At age 5 months, there is a striking 

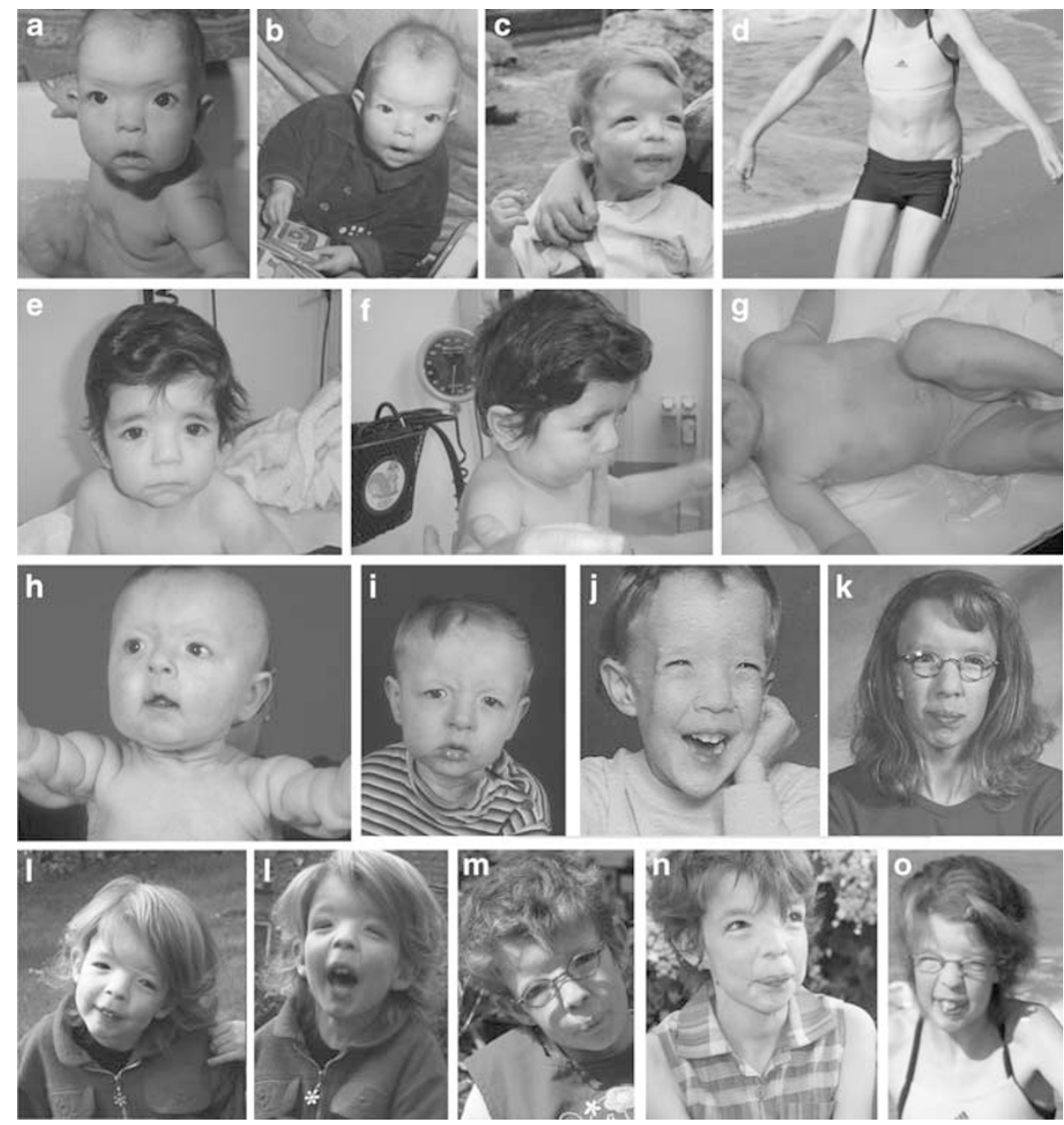

Figure 1 Patient 1: facial features with short palpebral fissures, midfacial hypoplasia, long phyltrum (a, b) and mild abdominal cutis laxa at the age of 14 months (a), facial features at 38 months demonstrating down-slanting palpebral fissures (c), 4 years (I), 9 years (m) and 10 years (n). Myopia, brittle hair and improving skin anomalies at the age of 11.5 years $(\mathbf{d}, \mathbf{o})$. Patient 2 : characteristic facial features $(\mathbf{e}, \mathbf{f})$ and mild skin abnormalities $(\mathbf{g})$ at the age of 16 months. Patient 6: midfacial hypoplasia at the age of 5 years (j), brittle hair and development of hypermetropia at the age of 16 years (k). Patient 7: abnormal fat distribution on the arms and abdominal cutis laxa at the age of 11 months (h), and characteristic face at the age of 4.5 years (i).

microcephaly (OFC $36 \mathrm{~cm}$ : <-4 SD), hypertelorism with down-slanting palpebral fissures, beaked nose with a broad nasal bridge, high, narrow palate and retrognathia (Figure $2 \mathrm{a}-\mathrm{d})$, joint laxity but no muscle hypotonia. Psychomotor development appears to be only mildly delayed.

Patient 6 was the first female child of healthy nonrelated parents. Generalized cutis laxa, brittle hair, microcephaly, large anterior fontanel $(6 \times 5 \mathrm{~cm})$, midfacial hypoplasia, anteverted nares, down-slanting palpebral fissures, epicanthal folds, highly arched palate and strabismus were noted after birth. The child had transient feeding difficulties and developed severe tonic-clonic seizures at the age of 14 years. Skin disease showed a continuous improvement (Figure $1 \mathrm{j}$ and $\mathrm{k}$ ), but at the age of 16 years she still has sparse, brittle hair and developed myopia (Figure $1 \mathrm{j}$ and $\mathrm{k}$ ). She was admitted to the hospital at the age of 5 years for a large cranial hematoma after minor trauma; however, no thrombocytopenia or coagulation defect could be confirmed. She has normal length and decreased weight $(<-2 \mathrm{SD})$ and her head circumference is in the lower normal range $(-1.5 \mathrm{SD})$. She is treated for a late onset absence epilepsy with combined antiepileptic therapy and has an IQ of 91.

Patient 7 was the second male child of a FrenchCanadian couple. Consanguinity was possible but not confirmed. He presented with microcephaly (OFC $30 \mathrm{~cm}$ : $<-2 \mathrm{SD})$, generalized cutis laxa, large anterior fontanel, high arched palate, hypotonia and facial dysmorphic features, including down-slanting palpebral fissures, anteverted nares and long philtrum. Cardiac ultrasound found a coarctation of the aorta with bicuspid aortic valve and atrio-ventricular septal defect. Abnormal lipid distribution became more apparent on the extremities (Figure 1h). Abdominal ultrasound was normal. At 4 years and 8 

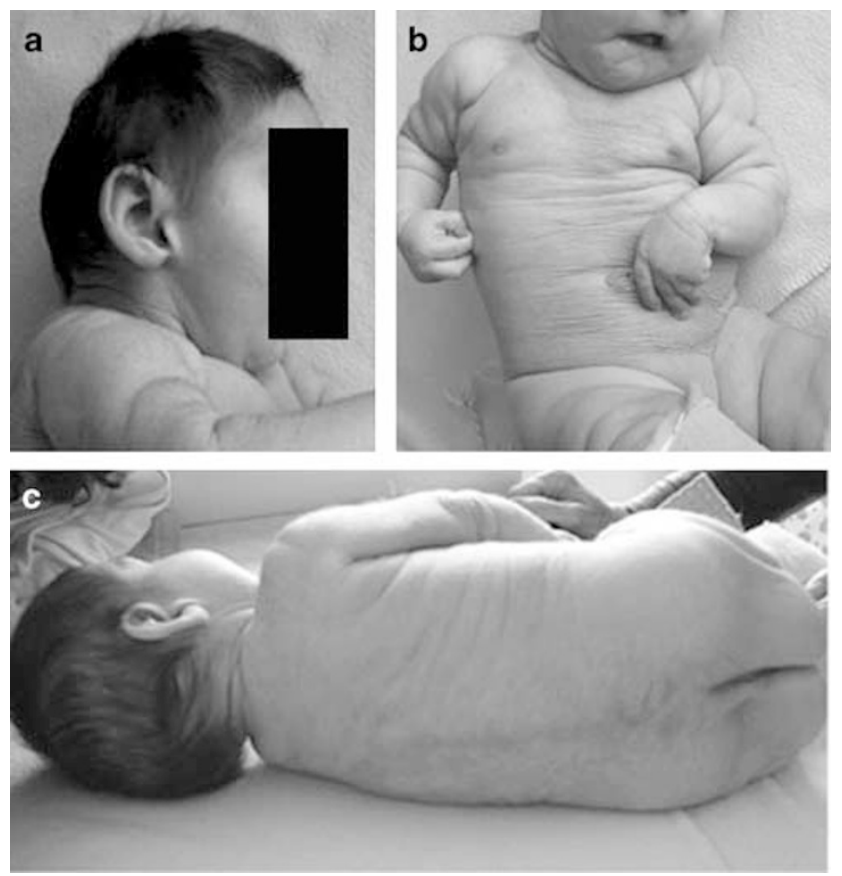

Figure 2 Facial features in patient 5 demonstrating microcephaly (a) retrognathia, long philtrum (b) and wrinkly skin with unequal fat distribution $(\mathbf{a}, \mathbf{b}, \mathbf{c})$ at the age of 16 months.

months the growth parameters show an age-appropriate weight and height with microcephaly (OFC $47 \mathrm{~cm}:<-2$ $\mathrm{SD})$. His psychomotor development is within normal limits but shows mild hypotonia. He developed a beaked nose and an abnormal fat distribution around the hips by a continuous improvement of the cutis laxa (Figure 1i).

\section{Results}

Skin biopsy was performed in patients 1, 2 and 3, demonstrating abnormal, broken, shortened and fuzzy elastic fiber structure with a significantly decreased amount of elastin by histology (Figure 4). Ophthalmologic evaluation showed a strabismus in patients $2,3,5$ and 6 , myopia ( $<-5$ diopters) was found in patients 1 and 6 , amblyopia with hypermetropia in patient 4 and hypermetropia in patient 7 . Echocardiogram was normal in patients $2,3,4,5$ and 6 . In patient 7 , a complex congenital cardiac anomaly was detected. EEG, BAEP and a cranial MRI/CT were normal in patients 1,5 and 6. No occipital horn formation was found. Multicystic frontal lesions were detected with reactive gliosis in patient 2 . Bilateral frontotemporal pachygyria/polymicrogyria, white matter anomalies, delayed myelinization and migration defects were observed in patient 3 and bilateral frontal focal polymicrogyria, in patient 7 . Sonography of the kidneys revealed a bilateral mild hydronephrosis in patient 5 . The uterus and ovaries could not be visualized in patient 5 . A radiograph of the skull showed poor mineralization of the neurocranium in patient 5 (Table 1).

Routine laboratory investigations were normal, except for a slightly elevated activity of alanine amino transferase (ALAT) $(90 \mathrm{U} / \mathrm{l}$, ref. $<50 \mathrm{U} / \mathrm{l})$ in patient 1 and aspartate aminotransferase (ASAT) $(86 \mathrm{U} / \mathrm{l}$, ref. <50 U/l) in patients 2 and 3, with normal activity of the gamma-glutamyl transferase. By screening investigation of coagulation, APTT was only mildly elevated in patient 3 (42s, ref. 25-39s).

Patients 1-7 (and the previously reported patients 8,9 and 10; see tables) all had an abnormal plasma transferrin isoform profile suggesting a defect in $\mathrm{N}$-glycan biosynthesis (Table 2). This is a so-called disialo type 2 profile $^{15}$ characterized by normal or only slightly elevated levels of asialo- and monosialo transferrin and increasingly higher levels of disialo- and trisialo transferrin. This strongly suggests a primary defect in the Golgi part of the N-glycan biosynthesis pathway. Plasma ApoC-III isofocusing showed a typical ApoC-III ${ }_{1}$ profile ${ }^{15}$ characterized by elevated levels of the monosialo ApoC-III isoform and decreased levels of its disialoform (Table 1). This profile has only been described in cutis laxa patients ${ }^{11,15}$ and in a patient with CDG type IIf (cytidine $5^{\prime}$ monophospho- $\mathrm{N}$-acetylneuraminic acid transporter defect ${ }^{17}$ ). This finding suggests a defect in mucin type core 1 O-glycan biosynthesis. Patients 1-10 form the biochemical group 6 described by Wopereis et al. ${ }^{15}$ The patients have a combined defect in $\mathrm{N}$ - and O-glycan biosynthesis.

In a postnatal blood sample taken at 2 months of age, transferrin isofocusing gave a normal result in patient 2 . The first abnormal result was obtained at 6 months of age in this patient. In this child, the ApoC-III isofocusing gave an abnormal result in all three plasma samples. A similar observation was made in patient 10 (case $3^{10}$ ), who had a normal plasma transferrin distribution but an abnormal ApoC-III profile at 3 months of age, while both tests gave an abnormal result at 3 years of age.

Karyotype was normal in all children. FISH 7q11.23 was normal in patients 1 and 6. FISH 22q11.2 and multisubtelomeric FISH were normal in patient 7 . Genome-wide homozygosity mapping in the three previously described and one of the here described consanguineous families (Table 1 and Figure 3), using the 10K and 250K Affymetrix single nucleotide polymorphism arrays, ${ }^{17}$ revealed a $3 \mathrm{MB}$ homozygous region on chromosome 12 (12q24.31), shared by all patients tested. Genetic material was available for molecular analysis in 9 out of 10 children (except for patient 2). Sequence analysis excluded mutations in the coding exons (including intron-exon boundaries) of B3GNT4, MPHOSPH9 and TMED2. Different missense and/or nonsense mutations were found by Kornak et $a l^{18}$ in all children, including the three patients described previously (patients 8,9 and 10), ${ }^{9}$ in the ATP6VOA2 gene. 
a

C

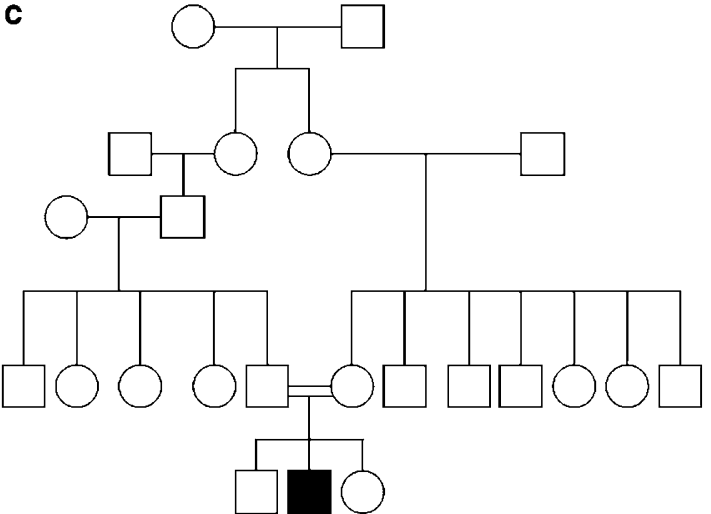

b

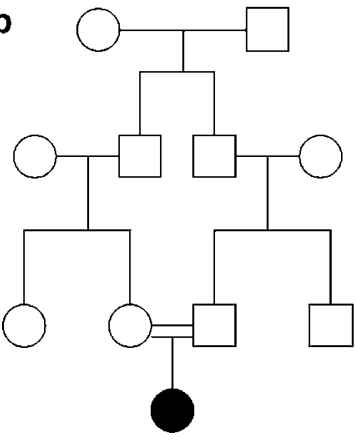

d

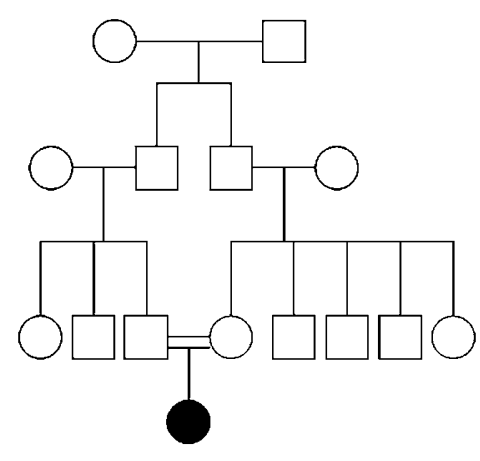

Figure 3 Consanguineous pedigrees of four probands with cutis laxa and a combined glycosylation disorder; (a), patient 1; (b), patient 2; (c), patient 5; (d), patient 4 .
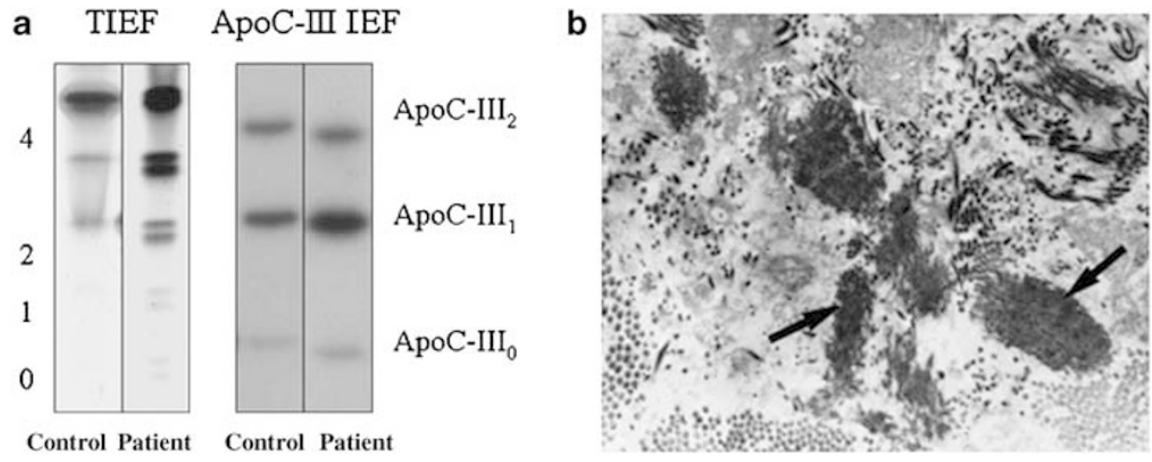

Figure 4 Abnormal transferrin pattern with decreased tetrasialotransferrin, increased hypoglycosylated tri-, di- and monosialylated transferrin isoforms and abnormal ApoC-III pattern with increased levels of ApoC- $-I_{1}$, decreased levels of ApoC-III ${ }_{2}$ and normal ApoC-III $I_{0}$ in patient 1 (a). Abnormal staining of elastin in the skin biopsy of patient 3 demonstrating frayed, fuzzy elastic fibers, reduced in number and density (b).

\section{Discussion}

Here we define the phenotype of the ARCL syndrome associated with a combined $\mathrm{N}$ - and O-linked glycan biosynthesis defect. All patients have generalized cutis laxa at birth, involving the face, trunk and extremities. Further specific findings include late closure of the fontanel, characteristic facial appearance with down-slanting palpebral fissures, short nose, small mouth and, in some of the children, joint anomalies (congenital hip dislocation, joint laxity and pes planus). These features have been described previously in the majority of patients with ARCL-II (MIM 219100). In our group, however, normal length and relative low body weight was found in most of the cases. Besides the late closure of the fontanel, no other skeletal or bone anomalies are reported by a full skeletal survey, except for decreased cranial mineralization and severe 
Table 2 Transferrin IEF and ApoC-III IEF results demonstrating the percentage of the glycosylated fraction in our patient cohort and in those reported previously

\begin{tabular}{|c|c|c|c|c|c|c|}
\hline & & & Transferrin & & & \\
\hline & Age in years & Monosialo & Disialo & Trisialo & ApoC-III ${ }_{1}$ & ApoC-III \\
\hline Patient 1 & 11 & 3.0 & 16 & 32 & 80 & 14 \\
\hline Patient 3 & 2 & 2.0 & 11 & 29 & 89 & 7 \\
\hline Patient 4 & 3 & 1.0 & 10 & 25 & 72 & 24 \\
\hline Patient 5 & 0.4 & 3.0 & 10 & 22 & 88 & 11 \\
\hline Patient 6 & 28 & 1.0 & 12 & 28 & 91 & 7 \\
\hline Patient $9^{*}$ & 2 & 5.0 & 14 & 23 & 76 & 14 \\
\hline Patient $10^{*}$ & 3 & 3.0 & 12 & 27 & 86 & 8 \\
\hline Reference va & & & & & & \\
\hline All ages & & $0-3.0$ & $1.0-6.0$ & $3.0-16$ & & \\
\hline $\begin{array}{l}0-1 \text { year } \\
>1 \text { year }\end{array}$ & & & & & $\begin{array}{l}34-59 \\
33-67\end{array}$ & $\begin{array}{l}40-62 \\
27-60\end{array}$ \\
\hline
\end{tabular}

*Morava et al. ${ }^{9}$

Table 3 Differential diagnosis of autosomal recessive cutis laxa

\begin{tabular}{lccc}
\hline & ARCL- I & ARCL-II & ARCL-III \\
\hline Facial dysmorphism & +++ & +++ & +++ \\
Hernias & +++ & +++ & ++ \\
Joint laxity & + & +++ & +++ \\
Hypotonia & + & +++ & - \\
Congenital hip dislocation & + & ++ & - \\
Significant CV abnormalities & +++ & + & - \\
Bladder diverticula & ++ & - & - \\
Emphysema & +++ & - & - \\
IUGR & - & +++ & +++ \\
Delayed motor development & - & +++ & +++ \\
Mental retardation & - & ++ & ++ \\
Eye anomalies & - & +++ & +++ \\
Large anterior fontanel & - & +++ & ++ \\
Microcephaly & - & +++ & $?$ \\
Seizures & - & ++ & $?$ \\
Intracranial anomaly & - & ++ & $?$ \\
Athetoid movements & - & - & +++ \\
Corneal clouding & - & - & +++ \\
\hline
\end{tabular}

$A R C L$, autosomal recessive cutis laxa; CV, cardiovascular; IUGR, intrauterine growth retardation.

+++ , common finding; ++ , several case reports found;,$+ 1-2$ case reports found; - , not present.

caries in two females. None of the patients developed occipital horns, a characteristic feature in the X-linked form of syndromic cutis laxa (MIM 304150).

There is a strikingly changing, evolving phenotype of the skin anomalies with diminishing of the cutis laxa with age. Most children have no apparent fat distribution anomalies at birth, but with the improvement of the wrinkled skin the abnormal fat distribution around the buttock and the hips becomes more and more apparent.

Interestingly, in this subtype of congenital disorder of glycosylation, systemic involvement, including elevated liver enzyme activities and slightly abnormal coagulation in some of the patients, is very mild. No apparent hypoglycemia, endocrine, cardiac or renal involvement has been noted in the patients. Severe pulmonary and cardiovascular involvement described in ARCL associated with fibulin-4 (FBLN $\left.4^{3}\right)$ and fibulin-5 (FBLN $\left.5^{4}\right)$ mutations were also absent in our patients.

In a few cases, microcephaly is present already at birth, and in some children it progressed further in the course of the disease. Progressive microcephaly is quite unique in view of the finding of a large and persistent open fontanel. Congenital central nervous system anomalies are found only in the minority of the patients. Epilepsy (late onset seizures) occurred in 1 out of the 7 children described here. In the total number of 10 patients, including the cases previously described, ${ }^{9,11}$ sensorineural hearing impairment was noted only in patient 8 (Table 1 ). Developmental delay can be observed in the majority of the children, partially due to muscle hypotonia and to some degree hyperflexible joints. While muscle hypotonia can lead to secondary delays in motor skill acquisition, in the view of the frequent occurrence of microcephaly, the main contributor to the mild-moderate developmental delay is most likely central in origin. Mental retardation was present in most older cases in our patient cohort.

The phenotypic spectrum in our patients resembles ARCL type II (ARCL-II, MIM 219100), also known as cutis laxa with growth and developmental delay (Table 3). This type of cutis laxa can be readily distinguished from ARCL type I, which is commonly associated with severe cardiopulmonary lesions, including infantile emphysema and supravalvular aortic stenosis, and is caused by mutations in the FBLN5 gene. ${ }^{4,19}$ There are many overlapping features regarding the phenotype in ARCL type III (MIM 219150) and our patients. Although both groups show a late closure of the anterior fontanel, the facial features are quite 
different, demonstrating a somewhat older, almost progeroid appearance in ARCL type III (De Barsy syndrome ${ }^{20}$ ). Other diagnostic signs such as athetoid movements and corneal opacification can distinguish ARCL type III from both ARCL-II and ARCL-I (Table 3).

Homozygosity mapping in patients from consanguineous parents showed a common homozygous region on chromosome 12. Direct sequencing of the ATP6VOA2 gene resulted in the detection of mutations in four patients described here with the phenotype of ARCL-II and CDG type II. ${ }^{18}$

Still, it is intriguing that some patients with ARCL-II with the same phenotype do not show glycosylation anomalies supporting genetic heterogeneity in ARCL syndrome.

The phenotypic variability regarding growth and development could be the consequence of consanguinity; however, it still highlights the usefulness of glycosylation studies in the diagnosis of ARCL-II. It remains open for further investigations whether ARCL-II is heterogeneous and may occur both with and without glycosylation abnormalities. In our patient group demonstrating a distinct clinical and biochemical phenotype, however, we were able to detect the same underlying genetic defect.

All of the children described in our report have a combined disorder of glycosylation with a characteristic TIEF pattern (CDG type II) and an abnormal isofocusing profile for core 1 mucin type O-glycan in blood, demonstrated by the abnormal results of the ApoC-III IEF. The pattern is different from the severe hypoglycosylation observed in COG gene defects, ${ }^{21,22}$ but there is an obvious increased level of the ApoC- $\mathrm{III}_{1}$ fraction and decreased levels of ApoC-III 2 .

One should emphasize the possibility of missing the diagnosis of CDG syndrome in young infants with congenital cutis laxa by screening for glycosylation disorders by the TIEF method. In patient 2 , the results of the TIEF were normal at the age of 4 months and became diagnostic only months later. Fortunately, the ApoC-III IEF pattern was abnormal at an early stage of the disease, leading to the correct diagnosis. We had the same experience in one of our patients reported previously. ${ }^{10,11}$

Here we define an ARCL syndrome with a combined congenital defect of $\mathrm{N}$ - and O-linked glycosylation. We suggest evaluating the protein glycosylation status in all children with congenital wrinkled skin or cutis laxa, especially with characteristic facial features, late closure of the fontanel, variable central nervous system involvement and developmental delay.

\section{Acknowledgements}

This work was supported by grants from EUROGLYCANET contract number 512131 and NIH grants HLO73703 and HLO84922. We are thankful for the support of $H$ van Bokhoven, M Janssen, A Vogt and $L$ Berendsen regarding the linkage and sequence analysis.

\section{References}

1 Hashimoto K, Kanzaki T: Cutis laxa. Ultrastructural and biochemical studies. Arch Dermatol 1975; 111: 861-873.

2 Reisner SH, Seelenfreund M, Ben-Bassat M: Cutis laxa associated with severe intrauterine growth retardation and congenital dislocation of the hip. Acta Paediatr Scand 1971; 60: 357-360.

3 Hucthagowder V, Sausgruber N, Kim KH, Angle B, Marmorstein LY, Urban Z: Fibulin-4: a novel gene for an autosomal recessive cutis laxa syndrome. Am J Hum Genet 2006; 78: 1075-1080.

4 Loeys B, Van Maldergem L, Mortier G et al: Homozygosity for a missense mutation in fibulin-5 (FBLN5) results in a severe form of cutis laxa. Hum Mol Genet 2002; 11: 2113-2118.

5 Biver A, De Rijcke S, Toppet V, Ledoux-Corbusier M, Van Maldergem L: Congenital cutis laxa with ligamentous laxity and delayed development, Dandy-Walker malformation and minor heart and osseous defects. Clin Genet 1994; 45: 318-322.

6 Van Maldergem L, Ogur G, Yuksel M: Facial anomalies in congenital cutis laxa with retarded growth and skeletal dysplasia. Am J Med Genet 1989; 32: 265.

7 Gruenewald S, Matthijs G, Jaeken J: Congenital disorders of glycosylation: a review. Pediatr Res 2002; 52: 618-624.

8 Wopereis S, Gruenewald S, Morava E et al: Apolipoprotein C-III isofocusing in the diagnosis of genetic defects in O-glycan biosynthesis. Clin Chem 2003; 49: 1839-1845.

9 Morava E, Wopereis S, Coucke $\mathrm{P}$ et al: Defective protein glycosylation in patients with cutis laxa syndrome. Eur J Hum Genet 2005; 13: 414-421.

10 Wopereis S, Morava E, Gruenewald S et al: A combined defect in the biosynthesis of N- and O-glycans in patients with cutis laxa and neurological involvement: the biochemical characteristics. Biochim Biophys Acta 2005; 1741: 156-164.

11 Morava E, Willemsen MA, Wopereis S et al: High myopia and congenital myopathy with partial pachygyria in cutis laxa syndrome. Eur J Ophthalmol 2006; 16: 190-194.

12 Beltran-Valero de Bernabe D, Currier S, Steinbrecher A et al: Mutations in the O-mannosyltransferase gene POMT1 give rise to the severe neuronal migration disorder Walker-Warburg syndrome. Am J Hum Genet 2002; 71: 1033-1043.

13 van Reeuwijk J, Janssen M, van den Elzen C et al: POMT2 mutations cause alpha-dystroglycan hypoglycosylation and Walker-Warburg syndrome. J Med Genet 2005; 42: 907-912.

14 van Reeuwijk J, Brunner HG, van Bokhoven H: Glyc-O-genetics of Walker-Warburg syndrome. Clin Genet 2005; 67: 281-289.

15 Wopereis S, Morava E, Gruenewald S et al: Patients with unsolved congenital disorders of glycosylation type II can be subdivided in six distinct biochemical groups. Glycobiology 2005; 15: 1312-1319.

16 Wopereis S, Gruenewald S, Huijben KM et al: Transferrin and apolipoprotein C-III isofocusing are complementary in the diagnosis of $\mathrm{N}$ - and O-glycan biosynthesis defects. Clin Chem 2007; 53: 180-187.

17 Woods CG, Cox J, Springell K et al: Quantification of homozygosity in consanguineous individuals with autosomal recessive disease. Am J Hum Genet 2006; 78: 889-896.

18 Kornak U, Reynders E, Dimopoulou K et al: Mutations in the a2subunit of the v-type H+-ATPase impair glycosylation in the Golgi apparatus and cause autosomal recessive cutis laxa type 2, 2007. Nature Genetics, in press.

19 Elahi E, Kalhor R, Banihosseini SS et al: Homozygous missense mutation in fibulin-5 in an Iranian autosomal recessive cutis laxa pedigree and associated haplotype. J Invest Dermatol 2006; 126: 1506-1509.

20 de Barsy AM, Moens E, Dierckx L: Dwarfism, oligophrenia and degeneration of the elastic tissue in skin and cornea. A new syndrome? Helv Paediatr Acta 1968; 23: 305-313.

$21 \mathrm{Wu}$ X, Steet RA, Bohorov O et al: Mutation of the COG complex subunit gene COG7 causes a lethal congenital disorder. Nat Med 2004; 10: 518-523.

22 Morava E, Zeevaert R, Korsch E et al: A common mutation in the COG7 gene with a consistent phenotype including microcephaly, adducted thumbs, growth retardation, VSD and episodes of hyperthermia. Eur J Hum Genet 2007; 15: 638-645. 